\title{
C-COMPACTNESS MODULO AN IDEAL
}

\author{
M. K. GUPTA AND T. NOIRI \\ Received 24 January 2006; Revised 30 March 2006; Accepted 4 April 2006
}

We investigate the concepts of quasi- $H$-closed modulo an ideal which generalizes quasi$H$-closedness and $C$-compactness modulo an ideal which simultaneously generalizes $C$ compactness and compactness modulo an ideal. We obtain a characterization of maximal $C$-compactness modulo an ideal. Preservation of $C$-compactness modulo an ideal by functions is also investigated.

Copyright (c) 2006 Hindawi Publishing Corporation. All rights reserved.

\section{Introduction}

In the present paper, we consider a topological space equipped with an ideal, a theme that has been treated by Vaidyanathaswamy [15] and Kuratowski [6] in their classical texts. An ideal $\mathscr{I}$ on a set $X$ is a nonempty subset of $P(X)$, the power set of $X$, which is closed for subsets and finite unions. An ideal is also called a dual filter. $\{\phi\}$ and $P(X)$ are trivial examples of ideals. Some useful ideals are (i) $\mathscr{I}_{f}$, the ideal of all finite subsets of $X$, (ii) $\Phi_{c}$, the ideal of all countable subsets of $X$, (iii) $\mathscr{I}_{n}$, the ideal of all nowhere dense subsets in a topological space $(X, \tau)$, and (iv) $\Phi_{s}$, the set of all scattered sets in $(X, \tau)$. For an ideal $\mathscr{I}$ on $X$ and $A \subset X$, we denote the ideal $\{I \cap A: I \in \mathscr{I}\}$ by $\mathscr{I}_{A}$.

A topological space $(X, \tau)$ with an ideal $\mathscr{I}$ on $X$ is denoted by $(X, \tau, \mathscr{I})$. For a subset $A \subseteq X, A^{*}(\mathscr{I}, \tau)$ (called the adherence of $A$ modulo an ideal $\mathscr{I}$ ) or $A^{*}(\mathscr{\Psi})$ or just $A^{*}$ is the set $\{x \in X: A \cap U \notin \mathscr{I}$ for every open neighborhood $U$ of $x\}$. $A^{*}(\mathscr{I}, \tau)$ has been called the local function of $A$ with respect to $\mathscr{S}$ in [6]. It is easy to see that (i) for the ideal $\{\phi\}$, $A^{*}$ is the closure of $A$, (ii) for the ideal $P(X), A^{*}$ is $\phi$, and (iii) for ideal $\mathscr{I}_{f}, A^{*}$ is the set of all $\omega$-accumulation points of $A$. For general properties of the operator $*$, we refer the readers to $[5,14]$.

Observe that the operator $\mathrm{cl}^{*}: P(X) \rightarrow P(X)$ defined by $\mathrm{cl}^{*}(A)=A \cup A^{*}$ is a Kuratowski closure operator on $X$ and hence generates a topology $\tau^{*}(\mathscr{S})$ or just $\tau^{*}$ on $X$ finer than $\tau$. As has already been observed, $\tau^{*}(\{\phi\})=\tau$ and $\tau^{*}(P(X))=$ the discrete topology. A description of open sets in $\tau^{*}(\mathscr{P})$ as given in Vaidyanathaswamy [15] is given in the following. 
Theorem 1.1. If $\tau$ is a topology and $\mathscr{I}$ is an ideal, both defined on $X$, then

$$
\beta=\beta(\tau, \mathscr{I})=\{V-I: V \in \tau, I \in \mathscr{I}\} \text { is a base for the topology } \tau^{*}(\mathscr{I}) \text { on } X \text {. }
$$

Ideals have been used frequently in the fields closely related to topology, such as real analysis, measure theory, and lattice theory. Some interesting illustrations of $\tau^{*}(\mathscr{\Psi})$ are as follows [5].

(1) If $\tau$ is the topology generated by the partition $\{\{2 n-1,2 n\}: n \in \mathbb{N}\}$ on the set $\mathbb{N}$ of natural numbers, then $\tau^{*}\left(\mathscr{I}_{f}\right)$ is the discrete topology.

(2) If $\tau$ is the indiscrete topology on a set $X$, then $\tau^{*}\left(\mathscr{I}_{f}\right)$ is the cofinite topology on $X$, and $\tau^{*}\left(\mathscr{I}_{c}\right)$ is the co-countable topology on $X$. If for a fixed point $p \in X, \Phi$ denotes the ideal $\{A \subset X: p \notin A\}$, then $\tau^{*}(\mathscr{I})$ is the particular point topology on $X$.

(3) For any topological space $(X, \tau), \tau^{*}\left(\mathscr{I}_{n}\right)$ is the $\tau^{\alpha}$ topology of Njasstad [10].

(4) If $\tau$ is the usual topology on the real line $\mathbb{R}$ and $\mathscr{S}$ is the ideal of all subsets of Lebesgue measure zero, then $\tau^{*}$-Borel sets are precisely the Lebesgue measurable sets of $\mathbb{R}$.

\section{Quasi-H-closed modulo an ideal space}

The concept of compactness modulo an ideal was introduced by Newcomb [9] and has been studied among others by Rancin [11], and Hamlett and Janković [3]. A space $(X, \tau)$ is defined to be compact modulo an ideal $\mathscr{I}$ on $X$ or just $(\mathscr{S})$ compact space if for every open cover $\mathcal{U}$ of $X$, there is a finite subfamily $\left\{U_{1}, U_{2}, \ldots, U_{n}\right\}$ such that $X-\bigcup_{i=1}^{n} U_{i} \in$ $\Phi$. In this section, we define quasi-H-closedness modulo an ideal and study some of its properties. In the process, we get some interesting characterizations of quasi-H-closed spaces.

Definition 2.1. Let $(X, \tau)$ be a topological space and $\mathscr{I}$ an ideal on $X . X$ is quasi-H-closed modulo $\mathscr{I}$ or just $(\mathscr{I})$ QHC if for every open cover $U$ of $X$, there is a finite subfamily $\left\{U_{1}, U_{2}, \ldots, U_{n}\right\}$ of $U$ such that $X-\bigcup_{i=1}^{n} \mathrm{cl}\left(U_{i}\right) \in \mathscr{I}$. Such a subfamily is said to be proximate subcover modulo $\mathscr{I}$ or just $(\mathscr{I})$ proximate subcover.

A subset $A$ of a topological space $(X, \tau)$ is said to be preopen [8] if $A \subset \operatorname{int}(\operatorname{cl}(A))$. The collection of all preopen sets of a space $(X, \tau)$ is denoted by $\mathrm{PO}(X)$. An ideal $\mathscr{S}$ of subsets of a topological space $(X, \tau)$ is said to be codense [1] if the complement of each of its members is dense. Note that an ideal $\mathscr{I}$ is codense if and only if $\mathscr{I} \cap \tau=\{\phi\}$. Codense ideals are called $\tau$-boundary ideals in [9]. An ideal $\mathscr{I}$ of subsets of a topological space $(X, \tau)$ is said to be completely codense [1] if $\Phi \cap \mathrm{PO}(X)=\{\phi\}$. Obviously, every completely codense ideal is codense. Note that if $(\mathbb{R}, \tau)$ is the set $\mathbb{R}$ of real numbers equipped with the usual topology $\tau$, then $\mathscr{I}_{c}$ is codense but not completely codense ideal. It is proved in [1] that an ideal $\mathscr{S}$ is completely codense if and only if $\mathscr{I} \subset \mathscr{I}_{n}$.

From the discussion of Section 1, the proof of the following theorem is immediate.

Theorem 2.2. For a space $(X, \tau)$, the following are equivalent:

(a) $(X, \tau)$ is quasi-H-closed; 
(b) $(X, \tau)$ is $(\{\phi\}) \mathrm{QHC}$;

(c) $(X, \tau)$ is $\left(\mathscr{I}_{f}\right) \mathrm{QHC}$;

(d) $(X, \tau)$ is $\left(\mathscr{I}_{n}\right) \mathrm{QHC}$;

(e) $(X, \tau)$ is $(\mathscr{I})$ QHC for every codense ideal $\Phi$.

The significance of condition in (e) may be seen by considering the set $\mathbb{R}$ of real numbers equipped with the usual topology $\tau$. If $A$ is a finite subset of $\mathbb{R}$ and $\mathscr{I}$ is the ideal of all subsets of $\mathbb{R}-A$, then $(\mathbb{R}, \tau)$ is $(\mathscr{I}) \mathrm{QHC}$, but not quasi- $H$-closed.

A family $\mathscr{F}$ of subsets of $X$ is said to have the finite-intersection property modulo an ideal $\mathscr{I}$ on $X$ or just $(\mathscr{I})$ FIP if the intersection of no finite subfamily of $\mathscr{F}$ is a member of $\mathscr{I}$. Recall that a subset in a space is called regular open if it is the interior of its own closure. The complement of a regular open set is called regular closed. It is proved in [12] that for completely codense ideal $\mathscr{I}$ on a space $(X, \tau)$, the collections of regular open sets of $(X, \tau)$ and $\left(X, \tau^{*}\right)$ are same. The following theorem contains a number of characterizations of $(\mathscr{I})$ QHC spaces. Since the proof is similar to that of a theorem in the next section, we omit it.

Theorem 2.3. For a space $(X, \tau)$ and an ideal $\Phi$ on $X$, the following are equivalent:

(a) $(X, \tau)$ is $(\mathscr{I}) \mathrm{QHC}$;

(b) for each family $\mathscr{F}_{F}$ of closed sets having empty intersection, there is a finite subfamily $\left\{F_{1}, F_{2}, F_{3}, \ldots, F_{n}\right\}$ such that $\bigcap_{i=1}^{n} \operatorname{int}\left(F_{i}\right) \in \mathscr{I}$;

(c) for each family $\mathscr{F}$ of closed sets such that $\{\operatorname{int}(F): F \in \mathscr{F}\}$ has $(\mathscr{I})$ FIP, one has $\cap\{F$ : $F \in \mathscr{F}\} \neq \phi$;

(d) every regular open cover has a finite $(\mathscr{I})$ proximate subcover;

(e) for each family $F_{F}$ of nonempty regular closed sets having empty intersection, there is a finite subfamily $\left\{F_{1}, F_{2}, F_{3}, \ldots, F_{n}\right\}$ such that $\bigcap_{i=1}^{n} \operatorname{int}\left(F_{i}\right) \in \mathscr{I}$;

(f) for each collection $\mathscr{F}$ of nonempty regular closed sets such that $\left\{\operatorname{int}(F): F \in \mathscr{F}_{\mathcal{F}}\right\}$ has $(\mathscr{I}) \mathrm{FIP}$, one has $\bigcap\{F: F \in \mathscr{F}\} \neq \phi$;

(g) for each open filter base $\mathscr{B}$ on $P(X)-\mathscr{I}, \bigcap\{\mathrm{cl}(B): B \in \mathscr{B}\} \neq \phi$;

(h) every open ultrafilter on $P(X)-\mathscr{I}$ converges.

It follows from a result in [13] that $\tau$ and $\tau^{*}(\mathscr{T})$ have the same regular open sets, where $\Phi$ is a completely codense ideal on $(X, \tau)$. In particular, if $U \in \tau^{*}$, then $\mathrm{cl}(U)=\mathrm{cl}^{*}(U)$. Using this observation along with the previous theorem, we have the following.

Theorem 2.4. Let $\Phi$ be a completely codense ideal on a space $(X, \tau)$. Then $(X, \tau)$ is $(\mathscr{I}) \mathrm{QHC}$ if and only if $\left(X, \tau^{*}\right)$ is $(\mathscr{I}) \mathrm{QHC}$.

Combining this result with Theorem 2.2, we have the following.

Corollary 2.5. Let $(X, \tau)$ be a space and $\Phi$ a completely codense ideal on $X$. Then the following are equivalent:

(a) $(X, \tau)$ is quasi-H-closed;

(b) $\left(X, \tau^{*}\right)$ is quasi-H-closed;

(c) $\left(X, \tau^{\alpha}\right)$ is quasi-H-closed.

The last equivalence follows because $\tau^{\alpha}=\tau^{*}\left(\mathscr{I}_{n}\right)$, where $\mathscr{I}_{n}$ is the ideal of nowhere dense sets in $X$. 


\section{C-compact modulo an ideal space}

In this section, we generalize the concepts of $C$-compactness of Viglino [16] and compactness modulo an ideal due to Newcomb [9] and Rancin [11]. A space $(X, \tau)$ is said to be $C$-compact if for each closed set $A$ and each $\tau$-open covering $U$ of $A$, there exists a finite subfamily $\left\{U_{1}, U_{2}, U_{3}, \ldots, U_{n}\right\}$ such that $A \subset \bigcup_{i=1}^{n} \operatorname{cl}\left(U_{i}\right)$.

Definition 3.1. Let $(X, \tau)$ be a topological space and $\mathscr{I}$ an ideal on $X .(X, \tau)$ is said to be $C$ compact modulo $\mathscr{I}$ or just $C(\mathscr{I})$-compact if for every closed set $A$ and every $\tau$-open cover $\mathcal{U}$ of $A$, there is a finite subcollection $\left\{U_{1}, U_{2}, U_{3}, \ldots, U_{n}\right\}$ such that $A-\bigcup_{i=1}^{n} \operatorname{cl}\left(U_{i}\right) \in \mathscr{I}$.

It follows from the definition that

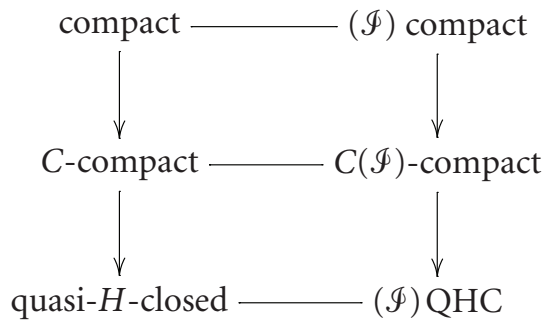

Also from the definition in Section 1, we have the following.

Theorem 3.2. For a space $(X, \tau)$, the following are equivalent:

(a) $(X, \tau)$ is C-compact;

(b) $(X, \tau)$ is $C(\{\phi\})$-compact;

(c) $(X, \tau)$ is $C\left(\mathscr{I}_{f}\right)$-compact.

Example 3.3. For $n$ and $m$ in the set $N$ of positive integers, let $Y$ denote the subset of the plane consisting of all points of the form $(1 / n, 1 / m)$ and the points of the form $(1 / n, 0)$. Let $X=Y \cup\{\infty\}$. Topologize $X$ as follows: let each point of the form $(1 / n, 1 / m)$ be open. Partition $N$ into infinitely many infinite-equivalence classes, $\left\{Z_{i}\right\}_{i=1}^{\infty}$. Let a neighborhood system for the point $(1 / i, 0)$ be composed of all sets of the form $G \cup F$, where

$$
\begin{aligned}
& G=\left\{\left(\frac{1}{i}, 0\right)\right\} \cup\left\{\left(\frac{1}{i}, \frac{1}{m}\right): m \geq k\right\}, \\
& F=\left\{\left(\frac{1}{n}, \frac{1}{m}\right): m \in Z_{i}, n \geq k\right\}
\end{aligned}
$$

for some $k \in N$. Let a neighborhood system for the point $\infty$ be composed of sets of the form $X \backslash T$, where

$$
T=\left\{\left(\frac{1}{n}, 0\right): n \in N\right\} \cup \bigcup_{i=1}^{k}\left\{\left(\frac{1}{i}, \frac{1}{m}\right): m \in N\right\} \cup\left\{\left(\frac{1}{n}, \frac{1}{m}\right): m \in Z_{i}, n \in N\right\}
$$

for some $k \in N$. It is shown in [16] that $X$ is a $C$-compact space which is not compact. In view of Theorem 3.2, such a space is $C\left(\mathscr{I}_{f}\right)$-compact, but not $\left(\mathscr{I}_{f}\right)$ compact. 
Example 3.4. Let $X=R^{+} \cup\{a\} \cup\{b\}$, where $R^{+}$denotes the set of nonnegative real numbers and $a, b$ are two distinct points not in $R^{+}$. Let $W(a)=\left\{V \subset X: V=\{a\} \cup \bigcup_{r=m}^{\infty}(2 r\right.$, $2 r+1)\}$, where $m$ is a nonnegative integer, be a neighborhood system for the point $a$. Let $W(b)=\left\{V \subset X: V=\{b\} \cup \bigcup_{r=m}^{\infty}(2 r-1,2 r)\right\}$, where $m$ is a nonnegative integer, be a neighborhood system for the point $b$. Let $R^{+}$, with the usual topology, be imbedded in $X$. Viglino [16] has shown that the space $X$ is not $C$-compact. If $A$ is a finite subset of $X$, then $(X, \tau)$ is $C(\mathscr{I})$-compact, where $\mathscr{I}$ is the ideal of all subsets of $X-A$.

In view of Examples 3.3 and 3.4, it is clear that the implications shown after Definition 3.1 are, in general, irreversible.

It is proved in [3] that if $(X, \tau)$ is quasi-H-closed and $\mathscr{I}$ is an ideal such that $\Phi_{n} \subset \mathscr{I}$, then $(X, \tau)$ is $(\mathscr{P})$ compact (and hence $C(\mathscr{I})$-compact).

Next, if $\left\{U_{1}, U_{2}, \ldots, U_{n}\right\}$ is a finite collection of open subsets such that $X-\bigcup_{i=1}^{n} \operatorname{cl}\left(U_{i}\right) \in$ $\Phi_{n}$, then $X-\bigcup_{i=1}^{n} \operatorname{cl}\left(U_{i}\right)=\phi$ because $\tau \cap \Phi_{n}=\{\phi\}$. But then $\operatorname{int}\left(\operatorname{cl}\left(X-\bigcup_{i=1}^{n} U_{i}\right)\right)=X-$ $\bigcup_{i=1}^{n} \operatorname{cl}\left(U_{i}\right)=\phi$ implies that $X-\bigcup_{i=1}^{n} U_{i} \in \mathscr{I}_{n}$. Therefore, a space $(X, \tau)$ is $\left(\mathscr{I}_{n}\right)$ compact if and only if it is $C\left(\mathscr{I}_{n}\right)$-compact. In view of this discussion, we have the following.

Theorem 3.5. For a space $(X, \tau)$, the following are equivalent:

(a) $(X, \tau)$ is quasi-H-closed;

(b) $(X, \tau)$ is $\left(\mathscr{I}_{n}\right) \mathrm{QHC}$;

(c) $(X, \tau)$ is $C\left(\mathscr{\Phi}_{n}\right)$-compact;

(d) $(X, \tau)$ is $\left(\Phi_{n}\right)$ compact.

A space $(X, \tau)$ is said to be Baire if the intersection of every countable family of open sets in $(X, \tau)$ is dense. It is noted in [5] that a space $(X, \tau)$ is Baire if and only if $\tau \cap \Phi_{m}=$ $\{\phi\}$, where $\Phi_{m}$ is the ideal of meager (first category) subsets of $(X, \tau)$. Thus, in view of the above theorem, a Baire space $(X, \tau)$ is $C\left(\Phi_{m}\right)$-compact if and only if it is quasi- $H$-closed.

We now give some characterizations of $C(\mathscr{I})$-compact spaces.

Theorem 3.6. Let $(X, \tau)$ be a space and let $\Phi$ be an ideal on $X$. Then the following are equivalent:

(a) $(X, \tau)$ is $C(\mathscr{I})$-compact;

(b) for each closed subset $A$ of $X$ and each family $F_{F}$ of closed subsets of $X$ such that $\bigcap\{F \cap A: F \in \mathscr{F}\}=\phi$, there exists a finite subfamily $\left\{F_{1}, F_{2}, F_{3}, \ldots, F_{n}\right\}$ such that $\bigcap\left(\operatorname{int}\left(F_{i}\right)\right) \cap A \in \mathscr{I}$;

(c) for each closed set $A$ and each family $\mathscr{F}$ of closed sets such that $\{\operatorname{int}(F) \cap A: F \in \mathscr{F}\}$ has $(\mathscr{I})$ FIP, one has $\cap\{F \cap A: F \in \mathscr{F}\} \neq \phi$;

(d) for each closed set $A$ and each regular open cover $U$ of $A$, there exists a finite subcollection $\left\{U_{1}, U_{2}, U_{3}, \ldots, U_{n}\right\}$ such that $A-\bigcup_{i=1}^{n} \operatorname{cl}\left(U_{i}\right) \in \Phi$;

(e) for each closed set $A$ and each family $\mathscr{F}$ of regular closed sets such that $\bigcap\{F \cap A: F \in$ $\mathscr{F}\}=\phi$, there is a finite subfamily $\left\{F_{1}, F_{2}, F_{3}, \ldots, F_{n}\right\}$ such that $\bigcap_{i=1}^{n}\left(\operatorname{int}\left(F_{i}\right)\right) \cap A \in$ $\mathscr{I}$

(f) for each closed set $A$ and each family $\mathscr{F}$ of regular closed sets such that $\{\operatorname{int}(F) \cap A$ : $F \in \mathscr{F}\}$ has $(\mathscr{I})$ FIP, one has $\bigcap\{F \cap A: F \in \mathscr{F}\} \neq \phi$;

(g) for each closed set $A$, each open cover $U$ of $X-A$ and each open neighborhood $V$ of $A$, there exists a finite subfamily $\left\{U_{1}, U_{2}, U_{3}, \ldots, U_{n}\right\}$ of $U$ such that $X-(V \cup$ $\left.\left(\bigcup_{i=1}^{n} \mathrm{cl}\left(U_{i}\right)\right)\right) \in \mathscr{I}$; 
(h) for each closed set $A$ and each open filter base $\mathscr{B}$ on $X$ such that $\{B \cap A: B \in \mathscr{B}\} \subset$ $P(X)-\mathscr{T}$, one has $\bigcap\{\mathrm{cl}(B): B \in \mathscr{B}\} \cap A \neq \phi$.

Proof. (a) $\Rightarrow(\mathrm{b})$. Let $(X, \tau)$ be $C(\mathscr{I})$-compact, $A$ a closed subset, and $\mathscr{F}$ a family of closed subsets with $\cap\{F \cap A: F \in \mathscr{F}\}=\phi$. Then $\{X-F: F \in \mathscr{F}\}$ is an open cover of $A$ and hence admits a finite subfamily $\left\{X-F_{i}: i=1,2, \ldots, n\right\}$ such that $A-\bigcup_{i=1}^{n} \operatorname{cl}\left(X-F_{i}\right) \in \mathscr{I}$. This set in $\mathscr{I}$ is easily seen to be $\bigcap_{i=1}^{n}\left\{\operatorname{int}\left(F_{i}\right) \cap A\right\}$.

(b) $\Rightarrow(\mathrm{c})$. This is easy to be established.

(c) $\Rightarrow$ (a). Let $A$ be a closed subset, let $U$ be an open cover of $A$ with the property that for no finite subfamily $\left\{U_{1}, U_{2}, U_{3}, \ldots, U_{n}\right\}$ of $\mathcal{U}$, one has $A-\bigcup_{i=1}^{n} \operatorname{cl}\left(U_{i}\right) \in \mathscr{I}$. Then $\{X-U: U \in \mathcal{U}\}$ is a family of closed sets. Since

$$
\bigcap_{i=1}^{n}\left\{X-\operatorname{cl}\left(U_{i}\right)\right\} \cap A=\bigcap_{i=1}^{n}\left\{A-\operatorname{cl}\left(U_{i}\right)\right\}=A-\bigcup_{i=1}^{n} \operatorname{cl}\left(U_{i}\right),
$$

the family $\{\operatorname{int}(X-U) \cap A: U \in \mathcal{U}\}$ has $(\mathscr{I})$ FIP. By the hypothesis $\bigcap\{(X-U) \cap A: U \in$ $\mathcal{U}\} \neq \phi$. But then $A-\cup\{U: U \in \mathcal{U}\} \neq \phi$, that is, $\boldsymbol{U}$ is not a cover of $A$, a contradiction.

$(\mathrm{d}) \Rightarrow(\mathrm{a})$. Let $A$ be a closed subset of $X$ and $U$ an open cover of $A$. Then $\{\operatorname{int}(\operatorname{cl}(U))$ : $U \in \mathcal{U}\}$ is a regular open cover of $A$. Let $\left\{\operatorname{int}\left(\operatorname{cl}\left(U_{i}\right)\right): i=1,2, \ldots, n\right\}$ be a finite subfamily such that $A-\bigcup_{i=1}^{n} \operatorname{cl}\left(\operatorname{int}\left(\operatorname{cl}\left(U_{i}\right)\right)\right) \in \mathscr{I}$. Since $U_{i}$ is open and for each open set $U, \operatorname{cl}(\operatorname{int}(\operatorname{cl}(U)))=\operatorname{cl}(U)$, we have $A-\bigcup_{i=1}^{n} \operatorname{cl}\left(U_{i}\right) \in \mathscr{I}$, which shows that $X$ is $C(\mathscr{I})$ compact.

(a) $\Rightarrow(d)$. This is obvious.

The proofs for $(\mathrm{d}) \Rightarrow(\mathrm{e}) \Rightarrow(\mathrm{f}) \Rightarrow(\mathrm{d})$ are parallel to $(\mathrm{a}) \Rightarrow(\mathrm{b}) \Rightarrow(\mathrm{c}) \Rightarrow(\mathrm{a})$, respectively.

$(\mathrm{a}) \Rightarrow(\mathrm{g})$. Let $A$ be a closed set, $V$ an open neighborhood of $A$, and $U$ an open cover of $X-A$. Since $X-V \subset X-A, \mathcal{U}$ is also an open cover of the closed set $X-V$.

Let $\left\{U_{1}, U_{2}, U_{3}, \ldots, U_{n}\right\}$ be a finite subcollection of $U$ such that $(X-V)-\bigcup_{i=1}^{n} \operatorname{cl}\left(U_{i}\right) \in$ $\Im$. However, the last set is $X-\left(V \cup\left\{\bigcup_{i=1}^{n} \operatorname{cl}\left(U_{i}\right)\right\}\right)$.

$(\mathrm{g}) \Rightarrow(\mathrm{a})$. Let $A$ be a closed subset of $X$ and $U$ an open covering of $A$. If $H$ denotes the union of members of $U$, then $F=X-H$ is a closed set and $X-A$ is an open neighborhood of $F$. Also $U$ is an open cover of $X-F$. By hypothesis, there is a finite subcollection $\left\{U_{1}, U_{2}, U_{3}, \ldots, U_{n}\right\}$ of $\mathcal{U}$ such that

$$
X\left((X-A) \cup\left\{\bigcup_{i=1}^{n} \mathrm{cl}\left(U_{i}\right)\right\}\right) \in \mathscr{I} .
$$

However, this set in $\mathscr{I}$ is nothing but $A-\bigcup_{i=1}^{n} \mathrm{cl}\left(U_{i}\right)$.

(a) $\Rightarrow(\mathrm{h})$. Suppose $A$ is a closed set and $\mathscr{B}$ is any open filter base on $X$ with $\{B \cap A$ : $B \in \mathscr{B}\} \subset P(X)-\mathscr{I}$. Suppose, if possible, $\bigcap\{\mathrm{cl}(B): B \in \mathscr{B}\} \cap A=\phi$. Then $\{X-\operatorname{cl}(B)$ : $B \in \mathscr{B}\}$ is an open cover of $A$. By the hypothesis, there exists a finite subfamily $\{X-$ $\left.\operatorname{cl}\left(B_{i}\right): i=1,2,3, \ldots, n\right\}$ such that $A-\bigcup_{i=1}^{n} \operatorname{cl}\left(X-\operatorname{cl}\left(B_{i}\right)\right)$ is in $\mathscr{I}$. However, this set is $A \cap$ $\left(\bigcap_{i=1}^{n} \operatorname{int}\left(\operatorname{cl}\left(B_{i}\right)\right)\right)$ and $A \cap\left(\bigcap_{i=1}^{n} B_{i}\right)$ is a subset of it. Therefore, $A \cap\left(\bigcap_{i=1}^{n} B_{i}\right) \in \mathscr{I}$. Since $\mathscr{B}$ is a filter base, we have a $B \in \mathscr{B}$ such that $B \subset \bigcap_{i=1}^{n} B_{i}$. But then $A \cap B \in \mathscr{I}$ which contradicts the fact that $\{B \cap A: B \in \mathscr{B}\} \subset P(X)-\mathscr{I}$.

$(\mathrm{h}) \Rightarrow(\mathrm{a})$. Suppose that $(X, \tau)$ is not $C(\mathscr{I})$-compact. Then there exist a closed subset $A$ of $X$ and an open cover $U$ of $A$ such that for any finite subfamily $\left\{U_{1}, U_{2}, U_{3}, \ldots, U_{n}\right\}$ 
of $\mathcal{U}$, we have $A-\bigcup_{i=1}^{n} \operatorname{cl}\left(U_{i}\right) \notin \mathscr{I}$. We may assume that $\mathcal{U}$ is closed under finite unions. Then the family $\mathscr{B}=\{X-\mathrm{cl}(U): U \in \mathcal{U}\}$ is an open filter base on $X$ such that $\{B \cap A$ : $B \in \mathscr{B}\} \subset P(A)-\mathscr{I}$. So, by the hypothesis, $\bigcap\{\mathrm{cl}(X-\operatorname{cl}(U)): U \in \mathcal{U}\} \cap A \neq \phi$. Let $x$ be a point in the intersection. Then $x \in A$ and $x \in \operatorname{cl}(X-\operatorname{cl}(U))=X-\operatorname{int}(\operatorname{cl}(U)) \subset X-U$ for each $U \in \mathcal{U}$. But this contradicts the fact that $U$ is a cover of $A$. Hence $(X, \tau)$ is $C(\mathscr{I})$ compact.

Next we characterize $C(\mathscr{I})$-compact spaces using some weaker forms of filter base convergence.

Definition 3.7. A filter base $\mathscr{B}$ is said to be $(\mathscr{T})$ adherent convergent if for every neighborhood $G$ of the adherent set of $\mathscr{B}$, there exists an element $B \in \mathscr{B}$ such that $(X-G) \cap B \in \mathscr{I}$. Clearly, every adherent convergent filter base is $(\mathscr{I})$ adherent convergent and a filter base is adherent convergent if and only if it is $(\{\phi\})$ adherent convergent.

Theorem 3.8. A space $(X, \tau)$ is $C(\mathscr{I})$-compact if and only if every open filter base on $P(X)-$ $\mathscr{I}$ is $(\mathscr{I})$ adherent convergent.

Proof. Let $(X, \tau)$ be $C(\mathscr{Y})$-compact and let $\mathscr{B}$ be an open filter base on $P(X)-\mathscr{I}$ with $A$ as its adherent set. Let $G$ be an open neighborhood of $A$. Then $A=\bigcap\{\operatorname{cl}(B): B \in$ $\mathscr{B}\}, A \subset G$, and $X-G$ is closed. Now $\{X-\operatorname{cl}(B): B \in \mathscr{B}\}$ is an open cover of $X-G$ and so by the hypothesis, it admits a finite subfamily $\left\{X-\operatorname{cl}\left(B_{i}\right): i=1,2,3, \ldots, n\right\}$ such that $(X-G)-\bigcup_{i=1}^{n} \operatorname{cl}\left(X-\operatorname{cl}\left(B_{i}\right)\right) \in \mathscr{I}$. But this implies $(X-G) \cap\left(\bigcap_{i=1}^{n} \operatorname{int}\left(\operatorname{cl}\left(B_{i}\right)\right)\right) \in \mathscr{I}$. However, $B_{i} \subset \operatorname{int}\left(\operatorname{cl}\left(B_{i}\right)\right)$ implies $(X-G) \cap\left(\bigcap_{i=1}^{n} B_{i}\right) \in \mathscr{I}$. Since $\mathscr{B}$ is a filter base and $B_{i} \in \mathscr{B}$, there is a $B \in \mathscr{B}$ such that $B \subset \bigcap_{i=1}^{n} B_{i}$. But then $(X-G) \cap B \in \mathscr{I}$ is required.

Conversely, let $(X, \tau)$ be not $C(\mathscr{I})$-compact, and let $A$ be a closed set, and $U$ an open cover of $A$ such that for no finite subfamily $\left\{U_{1}, U_{2}, U_{3}, \ldots, U_{n}\right\}$ of $\mathcal{U}$, one has $A-\bigcup_{i=1}^{n}$ $\operatorname{cl}\left(U_{i}\right) \in \Phi$. Without loss of generality, we may assume that $U$ is closed for finite unions. Therefore, $\mathscr{B}=\{X-\operatorname{cl}(U): U \in \mathcal{U}\}$ becomes an open filter base on $P(X)-\mathscr{I}$. If $x$ is an adherent point of $\mathscr{B}$, that is, if $x \in \bigcap\{\mathrm{cl}(X-\mathrm{cl}(U)): U \in \mathscr{U}\}=X-\bigcup\{\operatorname{int}(\operatorname{cl}(U))$ : $U \in \mathcal{U}\}$, then $x \notin A$, because $\mathcal{U}$ is an open cover of $A$ and for $U \in \mathcal{U}, U \subset \operatorname{int}(\operatorname{cl}(U))$. Therefore, the adherent set of $\mathscr{B}$ is contained in $X-A$, which is an open set. By the hypothesis, there exists an element $B \in \mathscr{B}$ such that $(X-(X-A)) \cap B \in \mathscr{I}$, that is, $A \cap$ $B \in \mathscr{I}$, that is, $A \cap(X-\operatorname{cl}(U)) \in \mathscr{I}$, that is, $A-\operatorname{cl}(U) \in \mathscr{I}$ for some $U \in \mathcal{U}$. This however contradicts our assumption. This completes the proof.

Herrington and Long [4] characterized $C$-compact spaces using $r$-convergence of filters and nets. We obtain similar results for $C(\mathscr{Y})$-compact spaces in the next definition.

Definition 3.9. Let $X$ be a space, $\phi \neq A \subset X$, and let $\mathscr{B}$ be a filter base on $A$. $\mathscr{B}$ is said to $r$-converge to $a \in A$ if for each open set $V$ in $X$ containing $a$, there is $B \in \mathscr{B}$ with $B \subset \mathrm{cl}(V)$. The filter base $\mathscr{B}$ is said to $r$-accumulate to $a$, if for each open set $V$ containing $a, \mathrm{cl}(V) \cap B \neq \phi$ for each $B \in \mathscr{B}$.

Similarly, a net $\varphi: D \rightarrow A \subset X$ is said to $r$-converge to $a \in A$ if for each open set $V$ containing $a$, there is a $b \in D$ such that $\varphi(c) \in \operatorname{cl}(V)$ for all $c \geq b$. $\varphi$ is said to $r$-accumulate to $a$ if for each open set $V$ containing $a$ and each $b \in D$, there is $c \in D$ with $c \geq b$ and $\varphi(c) \in \mathrm{cl}(V)$. 
It is known [4] that convergence (accumulation) for filter bases and nets implies $r$ convergence ( $r$-accumulation), but the converse is not true.

Theorem 3.10. For a space $(X, \tau)$ and an ideal $\Phi$ on $X$, the following are equivalent:

(a) $(X, \tau)$ is $C(\mathscr{I})$-compact;

(b) for each closed set $A$, each filter base $\mathscr{B}$ on $P(A)-\mathscr{I} r$-accumulates to some a $\in A$;

(c) for each closed set $A$, each maximal filter base $M$ on $P(A)-\mathscr{I} r$-converges to some $a \in A$;

(d) for each closed set $A$, each net $\varphi$ on $P(A)-\mathscr{I}$ r-accumulates to some $a \in A$.

Proof. (a) $\Rightarrow$ (b). Suppose there exist a closed set $A$ and a filter base $\mathscr{B}$ on $P(A)-\mathscr{I}$ which does not $r$-accumulate to any $a \in A$. Then for each $a \in A$, there exists an open set $U(a)$ containing $a$ and a $B(a) \in \mathscr{B}$ such that $B(a) \cap \operatorname{cl}(U(a))=\phi$. Then $\{U(a): a \in A\}$ is an open cover of the closed set $A$. By $(a)$, there exists a finite subcollection $\left\{U\left(a_{i}\right): i=\right.$ $1,2,3, \ldots, n\}$ such that $A-\bigcup_{i=1}^{n} \operatorname{cl}\left(U\left(a_{i}\right)\right) \in \mathscr{I}$. If $B \in \mathscr{B}$ is such that $B \subset \bigcap_{i=1}^{n} B\left(a_{i}\right)$, then $B \cap\left(A-\bigcup_{i=1}^{n} \mathrm{cl}\left(U\left(a_{i}\right)\right)\right) \in \mathscr{I}$, that is, $B-\bigcup_{i=1}^{n} \mathrm{cl}\left(U\left(a_{i}\right)\right) \in \mathscr{I}$. But the later set is just $B$, because $B \subset B\left(a_{i}\right)$ and $B\left(a_{i}\right) \cap \operatorname{cl}\left(U\left(a_{i}\right)\right)=\phi$ for each $i$. However, $B \in \mathscr{I}$ is a contradiction, because $B \in \mathscr{B}$ and $\mathscr{B} \subset P(A)-\mathscr{I}$.

(b) $\Leftrightarrow(c)$. This follows in view of parts (a), (b), and (c) of [4, Theorem 1].

(b) $\Rightarrow(\mathrm{a})$. If possible, let $X$ be not $C(\mathscr{I})$-compact. Then by Theorem 3.6(f), there exist a closed set $A$ and a collection $\mathscr{F}$ of regular closed sets with the property that for every finite subcollection $\left\{F_{1}, F_{2}, F_{3}, \ldots, F_{n}\right\}, \bigcap_{i=1}^{n} \operatorname{int}\left(F_{i}\right) \cap A \notin \mathscr{I}$, but $\bigcap\{F: F \in \mathscr{F}\} \cap A=\phi$. Now the collection of sets of the form $\bigcap_{i=1}^{n} \operatorname{int}\left(F_{i}\right) \cap A$ for all possible finite subfamilies $\left\{F_{1}, F_{2}, F_{3}, \ldots, F_{n}\right\}$ of $\mathscr{F}$ forms a filter base on $P(A)-\mathscr{I}$. By (b), this filter base $r$ accumulates to some $a \in A$, that is, for each open set $U(a)$ containing $a$ and for each $F \in \mathscr{F}, \operatorname{cl}(U(a)) \cap(\operatorname{int}(F) \cap A) \neq \phi$. However, $a \in A$ and $A \cap\{F: F \in \mathscr{F}\}=\phi$ imply that there is some $F=F(a) \in \mathscr{F}$ such that $a \notin F(a)$. Then $X-F(a)$ is an open set containing $a$ such that $\operatorname{cl}(X-F(a)) \cap(\operatorname{int}(F(a)) \cap A)=\phi$. This is a contradiction.

$(\mathrm{b}) \Leftrightarrow(\mathrm{d})$. This follows using standard arguments about nets and filters.

If in the above theorem, $A$ is replaced by the whole space $X$, we get the characterizations of $(\mathscr{I})$ QHC spaces. If in addition we consider completely codense ideal $\mathscr{I}$, we get the characterizations of quasi- $H$-closed spaces.

\section{4. $C(\mathscr{I})$-compact spaces and functions}

A function $f:(X, \tau)-(Y, \varsigma)$ is said to be $\theta$-continuous [2] at a point $x \in X$ if for every open set $V$ of $Y$ containing $f(x)$, there exists an open set $U$ of $X$ containing $x$ such that $f(\operatorname{cl}(U)) \subseteq \operatorname{cl}(V)$. A function $f:(X, \tau)-(Y, \varsigma)$ is said to be $\theta$-continuous if $f$ is $\theta$ continuous for every $x \in X$. The concept of $\theta$-continuity is weaker than that of continuity. An important property of $C$-compact spaces is that a continuous function from a $C$-compact space to a Hausdorff space is closed. We prove the following more general results.

Theorem 4.1. Let $f:(X, \tau, \mathscr{I})-(Y, \varsigma, \vartheta)$ be a $\theta$-continuous function, $(X, \tau, \mathscr{I}) C(\mathscr{I})$-compact, $(Y, \varsigma)$ Hausdorff, and $f(\mathscr{S}) \subseteq \vartheta$. Then $f(A)$ is $\varsigma^{*}(\vartheta)$-closed for each closed set $A$ of $X$. 
Proof. Let $A$ be any closed set in $X$ and $a \notin f(A)$. For each $x \in A$, there exists a $\varsigma$-open set $V_{y}$ containing $y=f(x)$ such that $a \notin \operatorname{cl}\left(V_{y}\right)$. Now because $f$ is $\theta$-continuous, there exists an open set $U_{x}$ containing $x$ such that $f\left(\operatorname{cl}\left(U_{x}\right)\right) \subseteq \operatorname{cl}\left(V_{y}\right)$. The family $\left\{U_{x}: x \in\right.$ $A\}$ is an open cover of $A$. Therefore, there exists a finite subfamily $\left\{U_{x_{i}}: i=1,2, \ldots, n\right\}$ such that $A-\bigcup_{i=1}^{n} \mathrm{cl}\left(U_{x_{i}}\right) \in \mathscr{I}$. But then $f\left(A-\bigcup_{i=1}^{n} \operatorname{cl}\left(U_{x_{i}}\right)\right) \in f(\mathscr{I}) \subseteq \vartheta$, that is, $f(A)-$ $f\left(\bigcup_{i=1}^{n} \operatorname{cl}\left(U_{x_{i}}\right)\right) \in f(\mathscr{I}) \subseteq \vartheta$ because $f(\mathscr{I})$ is also an ideal. Hence $f(A)-\left(\bigcup_{i=1}^{n} \operatorname{cl}\left(V_{y_{i}}\right)\right) \in$ $f(\mathscr{I}) \subseteq \vartheta$. Now $a \notin \operatorname{cl}\left(V_{y_{i}}\right)$ for any $i$ implies that $a \in Y-\bigcup_{i=1}^{n} \operatorname{cl}\left(V_{y_{i}}\right)$ which is open in $(Y, \varsigma)$ and $\left(Y-\bigcup_{i=1}^{n} \mathrm{cl}\left(V_{y_{i}}\right)\right) \cap f(A)=f(A)-\bigcup_{i=1}^{n} \mathrm{cl}\left(V_{y_{i}}\right) \in f(\mathscr{I}) \subseteq \vartheta$. Hence $a \notin(f(A)) *$ $(\sigma, \vartheta)$. Thus $(f(A))^{*}(\sigma, \vartheta) \subset f(A)$ and so $f(A)$ is $\varsigma^{*}(\vartheta)$-closed.

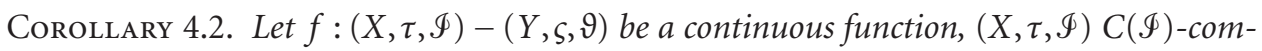
pact, $(Y, \varsigma)$ Hausdorff, and $f(\mathscr{Y}) \subseteq \vartheta$. Then $f(A)$ is $\varsigma^{*}(\vartheta)$-closed for each closed set $A$ of $X$.

Theorem 4.3. Let $f:(X, \tau, \mathscr{I})-(Y, \varsigma, \vartheta)$ be a continuous surjection, $(X, \tau, \mathscr{I}) C(\mathscr{I})$-compact, and $f(\mathscr{I}) \subseteq \vartheta$. Then $(Y, \varsigma, \vartheta)$ is $C(\vartheta)$-compact.

Proof. Let $A$ be any closed subset of $(Y, \varsigma)$ and $\left\{V_{\alpha}: \alpha \in \Lambda\right\}$ any open cover of $A$ by open sets in $Y$. Then $\left\{f^{-1}\left(V_{\alpha}\right): \alpha \in \Lambda\right\}$ is an open cover of $f^{-1}(A)$ which is closed in $X$. Hence, by the hypothesis, there exists a finite subcollection $\left\{f^{-1}\left(V_{\alpha_{i}}\right): i=1,2, \ldots, n\right\}$ such that $f^{-1}(A)-\bigcup_{i=1}^{n} \operatorname{cl}\left(f^{-1}\left(V_{\alpha_{i}}\right)\right) \in \mathscr{I}$. Since $f$ is continuous, $\operatorname{cl}\left(f^{-1}(B)\right) \subset f^{-1}(\operatorname{cl}(B))$ for every subset $B$ of $Y$. Hence we have $f^{-1}(A)-\bigcup_{i=1}^{n} f^{-1}\left(\operatorname{cl}\left(V_{\alpha_{i}}\right)\right)=f^{-1}\left(A-\bigcup_{i=1}^{n} \operatorname{cl}\left(V_{\alpha_{i}}\right)\right) \in \mathscr{I}$. Since $f$ is surjective, $A-\bigcup_{i=1}^{n} \operatorname{cl}\left(V_{\alpha_{i}}\right) \in f(\mathscr{F}) \subset \vartheta$. Hence $Y$ is $C(\vartheta)$-compact.

THeOREM 4.4. If the product space $\Pi X_{\alpha}$ of nonempty family of topological spaces $\left(X_{\alpha}, \tau_{\alpha}\right)$ is

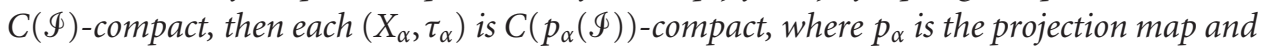
$\Phi$ is an ideal on $\Pi X_{\alpha}$.

Proof. This follows from Theorem 4.3.

\section{5. $C(\mathscr{F})$-compact spaces and subspaces}

In this section, we introduce three types of $C(\mathscr{I})$-compact subsets and use them to obtain new characterizations of $C(\mathscr{I})$-compact spaces and a characterization of maximal $C(\mathscr{I})$ compact spaces.

Definition 5.1. Let $(X, \tau)$ be a space and $\mathscr{S}$ an ideal on $X$. A subset $Y$ of $X$ is said to be $C(\mathscr{\Psi})$-compact if the subspace $\left(Y, \tau_{Y}\right)$ is $C(\mathscr{I})$-compact.

Some useful results about such subspaces are contained in the following theorem. The proofs are easy to establish.

Theorem 5.2. Let $(X, \tau)$ be a space and $\mathscr{I}$ an ideal on $X$. Then

(a) a subspace $Y$ is $C(\mathscr{S})$-compact if and only if it is $C\left(\mathscr{I}_{Y}\right)$-compact;

(b) a clopen subspace of a $C(\mathscr{I})$-compact space is $C(\mathscr{I})$-compact;

(c) if $Y$ is a regular closed subset of a $C(\mathscr{I})$-compact space $(X, \tau, \mathscr{S})$ and $\mathscr{\Phi}$ is codense, then $\left(Y, \tau_{Y}\right)$ is quasi-H-closed;

(d) a finite union of $C(\mathscr{I})$-compact subspaces of $X$ is $C(\mathscr{I})$-compact. 
Definition 5.3. A subset $Y$ of $(X, \tau)$ is said to be $C(\mathscr{I})$-compact relative to $\tau$ if every $\tau$-open cover of every relatively closed subset $A$ of $Y$ has a finite subfamily whose $\tau$-closures cover $A$ except a set in $\mathscr{I}$.

Some useful properties of such spaces are contained in the following.

Theorem 5.4. Let $(X, \tau)$ be a space and $\mathscr{I}$ an ideal on $X$. Then the following hold.

(a) A closed subspace of a $C(\mathscr{I})$-compact relative to $\tau$ subspace of $(X, \tau)$ is $C(\mathscr{Y})$-compact relative to $\tau$.

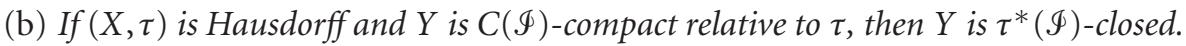

(c) If $Y$ is a $C(\mathscr{S})$-compact relative to $\tau$ subspace of $(X, \tau)$ and $f:(X, \tau)-(Z, \varsigma)$ is a continuous bijection, then $f(Y)$ is $C(f(\mathscr{I}))$-compact relative to $s$.

(d) $C(\mathscr{I})$-compactness relative to $\tau$ is contractive.

The following characterization of $C(\mathscr{T})$-compact spaces is obtained using $C(\mathscr{F})$-compact relative to $\tau$ subspaces. The proof is easy.

Theorem 5.5. A space $(X, \tau)$ with an ideal $\Phi$ is $C(\mathscr{S})$-compact if and only if every proper

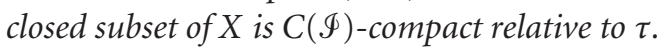

Definition 5.6. A subset $Y$ of a space $(X, \tau)$ is said to be closure $C(\mathscr{T})$-compact if for every $\tau_{Y}$-closed subset $K$ of $Y$ and every $\tau$-open cover $\boldsymbol{U}$ of $\mathrm{cl}(K)$, there is a finite subcollection $\left\{U_{1}, U_{2}, U_{3}, \ldots, U_{n}\right\}$ of $U$ such that $K-\bigcup_{i=1}^{n} \operatorname{cl}_{Y}\left(U_{i} \cap Y\right) \in \Phi$.

It is easy to see that closure $C(\mathscr{I})$-compactness is contractive.

Example 5.7. Since closed subsets of $C(\mathscr{I})$-compact spaces are not necessarily $(\mathscr{T}) \mathrm{QHC}$, a space $(X, \tau)$ which is $C(\mathscr{I})$-compact relative to $\tau$ may fail to be closure $C(\mathscr{I})$-compact. Moreover, $] 0,1]$ as a subspace of $[0,1]$ is closure $C(\mathscr{I})$-compact with $\mathscr{I}=\{\phi\}$, but not $C(\mathscr{I})$-compact relative to the usual topology. Thus the concepts of $C(\mathscr{I})$-compact relative to $\tau$ and closure $C(\mathscr{I})$-compact are independent concepts.

We now have the following characterization of $C(\mathscr{S})$-compact spaces.

Theorem 5.8. A space $(X, \tau)$ is $C(\mathscr{I})$-compact for an ideal $\mathscr{I}$ on $X$ if and only if every open subset of $X$ is closure $C(\mathscr{I})$-compact.

Proof. Let $(X, \tau)$ be $C(\mathscr{Y})$-compact and $Y$ an open subset of $X$. Let $K$ be a $\tau_{Y}$-closed subset of $Y$, and let $\mathcal{U}$ be a $\tau$-open cover of $\mathrm{cl}(K)$. Then there exists a finite subcollection $\left\{U_{1}, U_{2}, U_{3}, \ldots, U_{n}\right\}$ of $U$ such that $\mathrm{cl} K-\bigcup_{i=1}^{n} \mathrm{cl}\left(U_{i}\right) \in \mathscr{I}$. Since $Y$ is open, therefore, $\mathrm{cl}_{Y}(U \cap Y)=\mathrm{cl}(U) \cap Y$ and so, by hereditary property of $\mathscr{I}, K-\bigcup_{i=1}^{n} \mathrm{cl}_{Y}\left(U_{i} \cap Y\right) \in \mathscr{I}$. Thus $Y$ is closure $C(\mathscr{I})$-compact.

Conversely, let all open subsets of $X$ be closure $C(\mathscr{Y})$-compact. Let $K$ be a closed and $\mathcal{U}$ an open cover of $K$. Choose a $U_{0} \in \mathcal{U}$. Then $Y=X-\operatorname{cl}\left(U_{0}\right)$ is an open subset of $X$ and $K \cap Y$ is a $\tau_{Y}$-closed subset of $Y$. Moreover, $\mathcal{U}-\left\{U_{0}\right\}$ is an open cover of $\operatorname{cl}(K \cap Y)$. By the hypothesis, there exists a finite subcollection $\left\{U_{1}, U_{2}, U_{3}, \ldots, U_{n}\right\}$ of $\mathcal{U}-\left\{U_{0}\right\}$ such that $K \cap Y-\bigcup_{i=1}^{n} \operatorname{cl}_{Y}\left(U_{i} \cap Y\right) \in \mathscr{I}$. But then $K \cap Y-\bigcup_{i=1}^{n} \operatorname{cl}\left(U_{i}\right) \in \mathscr{I}$ as $\operatorname{cl}_{Y}\left(U_{i} \cap Y\right)=$ $\operatorname{cl}\left(U_{i}\right) \cap Y$ and $\mathscr{I}$ is hereditary. Therefore, $K-\bigcup_{i=0}^{n} \operatorname{cl}\left(U_{i}\right) \in \mathscr{I}$. Hence $(X, \tau)$ is $C(\mathscr{I})$ compact. 
Finally, we obtain a characterization of a maximal $C(\mathscr{\Psi})$-compact space. Recall that a space $(X, \tau)$ with property $P$ is said to be maximal $P$ if there is no topology $\sigma$ on $X$ which has property $P$ and is strictly finer than $\tau$. For a topological space $(X, \tau)$ and a subset $A$ of $X, \tau(A)=\{U \cup(V \cap A): U, V \in \tau\}$ is a topology called simple extension [7] of $\tau$ by $A$. $\tau(A)$ is strictly finer than $\tau$ if and only if $A \notin \tau$.

Theorem 5.9. A topological space $(X, \tau)$ is maximal $C(\mathscr{S})$-compact if and only if for every subset $A$ of $X$ such that $A$ is closure $C(\mathscr{I})$-compact and $X-A$ is $C(\mathscr{I})$-compact relative to $\tau$, one has $A \in \tau$.

Proof. First we assume that $(X, \tau)$ is maximal $C(\mathscr{\Psi})$-compact and that $A$ is a subset of $X$ satisfying the given conditions. First, we show that $(X, \tau(A))$ is $C(\mathscr{I})$-compact. Let $K$ be a $\tau(A)$-closed subset of $X$. Then $K=K_{1} \cup\left(K_{2} \cap(X-A)\right)$, where $K_{1}$ and $K_{2}$ are $\tau$-closed sets. Let

$$
\mathcal{U}=\left\{U_{\alpha} \cup\left(V_{\alpha} \cap A\right): U_{\alpha}, V_{\alpha} \in \tau, \alpha \in \Delta\right\}
$$

be a $\tau(A)$-open cover of $K$. Then $\nu=\left\{U_{\alpha}: \alpha \in \Delta\right\}$ is a $\tau$-open cover of $K \cap(X-A)=$ $\left(K_{1} \cup K_{2}\right) \cap(X-A)$. Since, by assumption, $X-A$ is $C(\mathscr{I})$-compact relative to $\tau$, we have a finite subcollection $\left\{U_{\alpha_{1}}, U_{\alpha_{2}}, U_{\alpha_{3}}, \ldots, U_{\alpha_{n}}\right\}$ of $\nu$ such that $K \cap(X-A)-\bigcup_{i=1}^{n} \operatorname{cl}\left(U_{\alpha_{i}}\right) \in \mathscr{I}$. Since $\tau(A)$ is finer than $\tau$, this subcollection is $\tau(A)$-open and $K \cap(X-A)-$ $\bigcup_{i=1}^{n} \mathrm{cl}_{\tau(A)}\left(U_{\alpha_{i}}\right) \in \mathscr{I}$. Next, $\mathcal{W}=\left\{U_{\alpha} \cup V_{\alpha}: \alpha \in \Delta\right\}$ is a $\tau$-open cover of $\operatorname{cl}(K \cap A)=$ $\operatorname{cl}\left(K_{1} \cap A\right)=\operatorname{cl}_{\tau(A)}\left(K_{1} \cap A\right)$ and therefore by assumption on $A$, there exists a finite subcollection $\left\{U_{\beta_{i}} \cup V_{\beta_{i}}: i=1,2, \ldots, k\right\}$ of $\mathcal{W}$ such that

$$
K_{1} \cap A-\bigcup_{i=1}^{k} \mathrm{cl}_{\tau_{A}}\left[\left(U_{\beta_{i}} \cup V_{\beta_{i}}\right) \cap A\right] \in \mathscr{I} .
$$

However, $\tau_{A}$, the restriction of $\tau$ to $A$, is nothing but $\tau(A) \mid A$, the restriction of $\tau(A)$ to A. Therefore,

$$
K_{1} \cap A-\bigcup_{i=1}^{k} \mathrm{cl}_{\tau(A) \mid A}\left[\left(U_{\beta_{i}} \cup V_{\beta_{i}}\right) \cap A\right] \in \mathscr{I} .
$$

Now $\left\{U_{\alpha_{i}} \cup\left(V_{\alpha_{i}} \cap A\right): i=1,2, \ldots, n\right\} \cup\left\{U_{\beta_{i}} \cup\left(V_{\beta_{i}} \cap A\right): i=1,2, \ldots, k\right\}$ is a finite $\tau(A)$ $(\mathscr{I})$ proximate cover of $K$ which is a subcover of $\mathcal{U}$. Thus the topology $\tau(A)$ on $X$ is also $C(\mathscr{\Phi})$-compact. However, by the maximality of $\tau$, we have $\tau(A)=\tau$. But then $A \in \tau$ as desired.

Conversely, let $(X, \tau)$ be not maximal $C(\mathscr{I})$-compact. Then there is a $C(\mathscr{I})$-compact topology $\sigma$ on $X$ which is strictly finer than $\tau$. Let $A \in \sigma-\tau$. Then $A$ is $\sigma$-closure $C(\mathscr{I})$ compact by Theorem 5.8. Since the property of closure $C(\mathscr{I})$-compact is carried over to coarser topologies, $A$ is $\tau$-closure $C(\mathscr{I})$-compact. Also $X-A$ is $C(\mathscr{I})$-compact relative to $\sigma$ and hence $C(\mathscr{Y})$-compact relative to $\tau$. By the hypothesis, then $A \in \tau$, a contradiction.

Remark 5.10. The readers can generalize the above concepts in bitopological spaces to unify various types of compactness. 


\section{References}

[1] J. Dontchev, M. Ganster, and D. A. Rose, Ideal resolvability, Topology and Its Applications 93 (1999), no. 1, 1-16.

[2] S. V. Fomin, Extensions of topological spaces, Annals of Mathematics. Second Series 44 (1943), 471-480.

[3] T. R. Hamlett and D. Janković, Compactness with respect to an ideal, Bollettino della Unione Matematica Italiana. B. Serie VII 4 (1990), no. 4, 849-861.

[4] L. L. Herrington and P. E. Long, Characterizations of C-compact spaces, Proceedings of the American Mathematical Society 52 (1975), 417-426.

[5] D. Janković and T. R. Hamlett, New topologies from old via ideals, The American Mathematical Monthly 97 (1990), no. 4, 295-310.

[6] K. Kuratowski, Topology. Vol. I, Academic Press, New York, 1966.

[7] N. Levine, Simple extensions of topologies, The American Mathematical Monthly 71 (1964), no. 1, $22-25$.

[8] A. S. Mashhour, M. E. Abd El-Monsef, and S. N. El-Deep, On precontinuous and weak precontinuous mappings, Proceedings of the Mathematical and Physical Society of Egypt (1982), no. 53, 47-53 (1983).

[9] R. L. Newcomb, Topologies which are compact modulo an ideal, Ph.D. thesis, University of California, Santa Barbara, California, 1967.

[10] O. Njàstad, On some classes of nearly open sets, Pacific Journal of Mathematics 15 (1965), 961970.

[11] D. V. Rancin, Compactness modulo an ideal, Soviet Mathematics. Doklady 13 (1972), 193-197.

[12] V. Renuka Devi, D. Sivaraj, and T. Tamizh Chelvam, Codense and completely codense ideals, Acta Mathematica Hungarica 108 (2005), no. 3, 197-205.

[13] P. Samuels, A topology formed from a given topology and ideal, Journal of the London Mathematical Society. Second Series 10 (1975), no. 4, 409-416.

[14] R. Vaidyanathaswamy, The localisation theory in set-topology, Proceedings of the Indian Academy of Sciences. Section A 20 (1944), 51-61.

[15] _ Set Topology, 2nd ed., Chelsea, New York, 1960.

[16] G. Viglino, C-compact spaces, Duke Mathematical Journal 36 (1969), no. 4, 761-764.

M. K. Gupta: Department of Mathematics, Faculty of Science, Ch. Charan Singh University, Meerut-250004, India

E-mail address: mkgupta2002@hotmail.com

T. Noiri: 2949-1, Shiokita-cho, Hinagu, Yatsushiro-shi, Kumamoto-ken 869-5142, Japan

E-mail address: t.noiri@nifty.com 


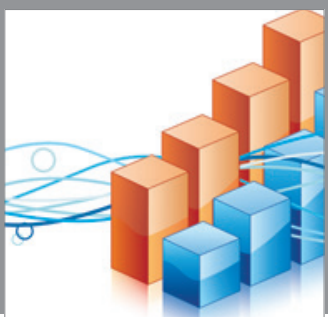

Advances in

Operations Research

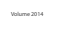

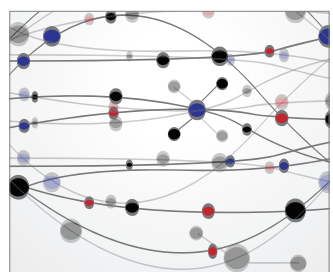

\section{The Scientific} World Journal
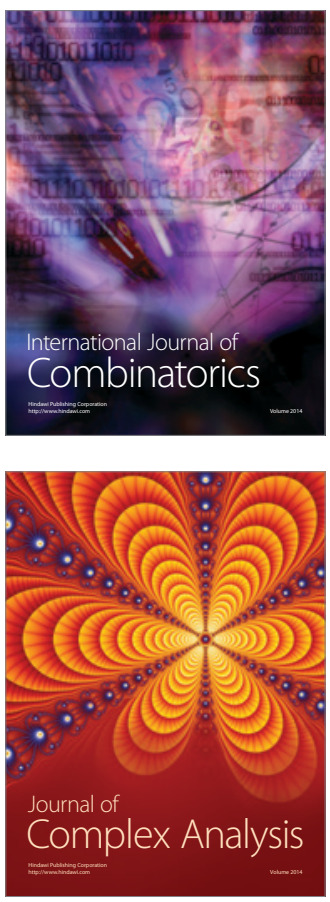

International Journal of

Mathematics and

Mathematical

Sciences
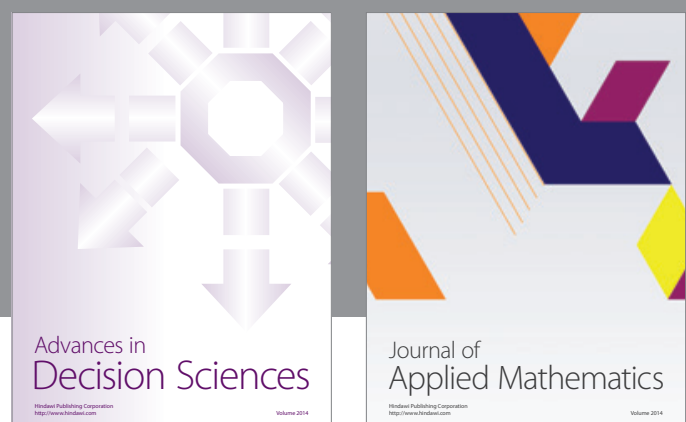

Journal of

Applied Mathematics
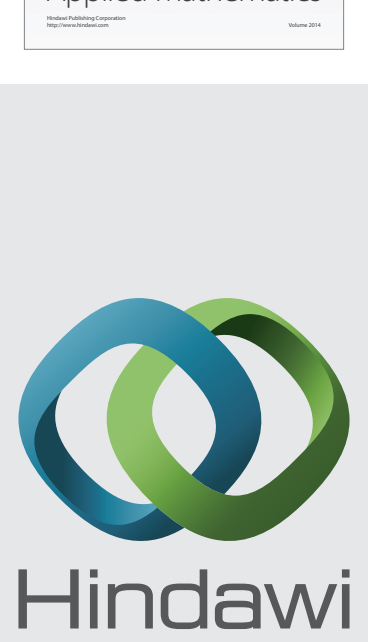

Submit your manuscripts at http://www.hindawi.com
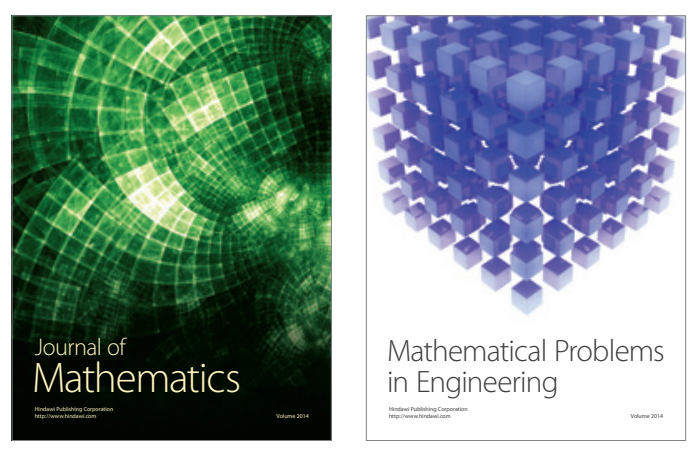

Mathematical Problems in Engineering
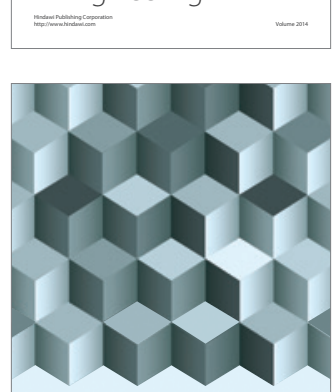

Journal of

Function Spaces
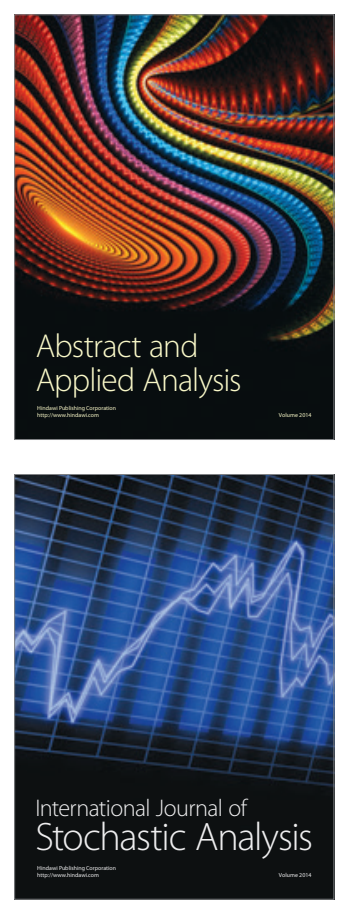

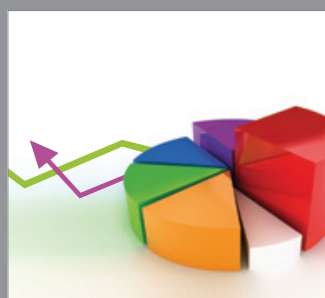

ournal of

Probability and Statistics

Promensencen
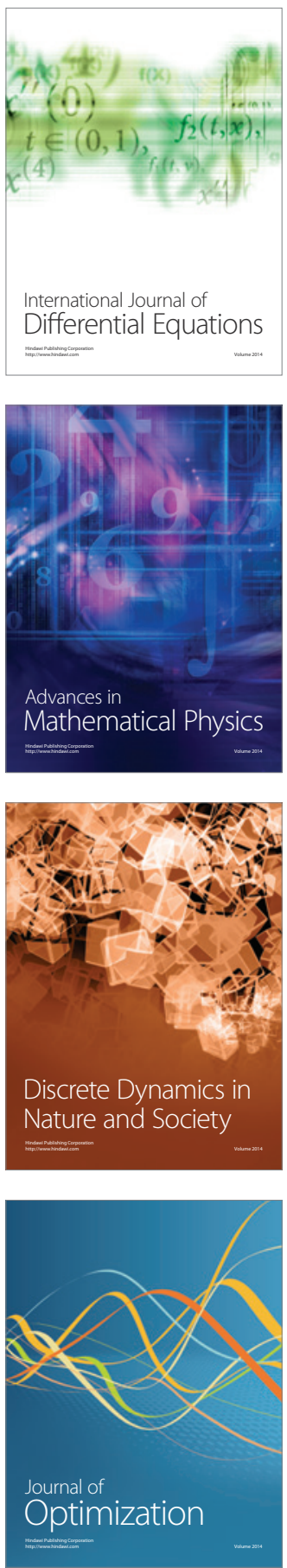